\title{
离子液体中溴钯化启动的烯炔烃串联的氧化杂环化反应研究
}

\author{
安艳妮李建晓*张振明李春生杨少容* \\ (华南理工大学化学与化工学院 广州 510640)
}

\begin{abstract}
摘要 室温条件下，以离子液体为溶剂，利用炔烃与烯烃衍生物发生串联氧化杂环化反应，以中等至优良产率 $(63 \%$ 84\%)合成系列官能团化的、饱和的五元含氧杂环化合物. 其结构均经 IR, ${ }^{1} \mathrm{H} N \mathrm{NR},{ }^{13} \mathrm{C}$ NMR 及 BRMS 确证. 该串联反 应具有反应条件温和、底物适用范围广、环境友好等优点, 可为具有饱和的五元含氧杂环化合物的天然产物及复杂药 物分子的合成提供简便的途径.
\end{abstract}

关键词 钯催化; 离子液体; 炔烃; 烯烃; 杂环化反应

\section{Bromopalladation Triggering the Cascade Oxidative Heterocyclization Reaction of Alkynes with Alkenes in lonic Liquids}

\author{
An, Yanni Li, Jianxiao* Zhang, Zhenming Li, Chunsheng Yang, Shaorong* \\ (School of Chemistry and Chemical Engineering, South China University of Technology, Guangzhou 510640)
}

\begin{abstract}
An efficient and practical palladium-catalyzed cascade oxidative heterocyclization has been developed to afford functionalized oxygen-containing five-membered heterocycles in moderate to good yields with high regio- and diastereoselectivities. Their structures were confirmed by IR, ${ }^{1} \mathrm{H}$ NMR, ${ }^{13} \mathrm{C}$ NMR and HRMS. Furthermore, the current methodology could also be conveniently applied to the synthesis of naturally occurring biologically active functionalized tetrahydrofurans and $\gamma$-lactones frameworks.
\end{abstract}

Keywords palladium-catalyzed; ionic liquids; alkynes; alkenes; heterocycles

杂环化学是有机合成化学重要的组成部分. 含氧、 氮等杂环化合物大多具有抗菌、消炎、抗病毒等生理活 性 ${ }^{[1]}$. 基于此, 大量的杂环化合物的合成方法被陆续报 道 ${ }^{[2]}$. 但是, 诸如繁琐的官能团保护和脱保护, 有毒、易 挥发的反应溶剂、较低的反应收率以及较差的选择性等, 是目前需要亟待解决的问题和缺憾. 过渡金属催化的不 饱和烯炔烃的偶联环化反应是实现杂环化合物构建的 一种构思巧妙、设计多样化的合成策略, 利用过渡金属 和不饱和烯炔烃的配位作用来实现烯炔烃的活化, 进而 通过系列串联反应以较高的收率和选择性实现各种杂 环体系的构建. ${ }^{[3]}$ 因此, 发展温和、绿色的催化反应体系 构建具有潜在生物活性的杂环骨架是有机合成化学和 绿色有机化学研究的热点课题 ${ }^{[4]}$.

另外, 五元氧杂环结构单元广泛存在于天然产物、 生物活性分子和药物分子中. 含有该结构骨架的衍生物
大多具有抗肿瘤、抗癌、抗菌等生理和药理活性 ${ }^{[5]}$. 因 此，该类型结构骨架的高效、高选择性地构筑受到国内 外有机合成研究者愈来愈多的关注和重视 ${ }^{[6]}$. 目前, 过 渡金属催化的不饱和烃的串联氧化、杂环化反应是构建 该结构骨架较为有效的合成策略. 比如, 陆熙炎课题组 报道了分子内烯炔类底物的氧化、杂环化反应 ${ }^{[7]}$, 并且 成功地实现了分子间炔酸和烯酫的杂环化反应合成系 列五元内酯化合物 ${ }^{[8]}$; Aurrecoechea 课题组 ${ }^{[9]}$ 报道了联三 烯醇与含有吸电子基的烯烃发生亲核氧钯化启动的偶 联反应, 合成四取代的呋喃衍生物; 进而, 该课题组 ${ }^{[10]}$ 以邻羟基苯乙炔衍生物和缺电子的烯烃发生偶联反应, 亦能得到多取代的苯并呋喃化合物. 此外, Wolfe 课题 组 ${ }^{[11]}$ 在构建五元氧杂环化合物方面做了大量的研究工 作. 近年来, 我们课题组 ${ }^{[12 \sim 15]}$ 在常规有机溶剂, 比如乙 腈作溶剂的条件下成功实现了亲核氯钯化启动的烯炔

*E-mail: lisryang@scut.edu.cn

Received March 27, 2016; revised April 14, 2016; published online May 17, 2016

Project supported by the National Natural Science Foundation of China (No. 21502055), the Fundamental Research Funds for the Central Universities (No. 2015ZM150) and China Postdoctoral Science Foundation (No. 2015M572303).

项目受国家自然科学基金(No. 21502055), 中央高校基本科研业务费(No. 2015ZM150)和中国博士后科学基金(No. 2015M572303)资助. 
烃串联的氧化杂环化反应, 高效、高选择性地构建系列 $\gamma$-丁内酯和四氢呋喃衍生物. 同时, 当以绿色溶剂离子 液体为介质, 我们 ${ }^{[16]}$ 发展了以氯钯化启动的烯烃的串 联的双官能团化反应，成功地构建系列饱和的 $\beta$ 或 $\gamma$-丁 内酯类化合物.

因此, 我们课题组在前期工作的研究基础上 ${ }^{[16,17]}$, 进一步以炔烃和系列烯烃为底物, 在离子液体中研究了 它们的串联碳醚化和碳酯化反应. 结果表明, 以 $\mathrm{PdBr}_{2}$ 作催化剂, $\mathrm{CuBr}_{2}$ 作氧化剂, 离子液体 $\left[\mathrm{C}_{2} \mathrm{O}_{2} \mathrm{mim}\right] \mathrm{Br}$ 作 溶剂, 室温条件下能以较高产率(63\% 84\%)简捷、高选 择性地合成系列官能团化的五元氧杂环化合物(Eq. 1).

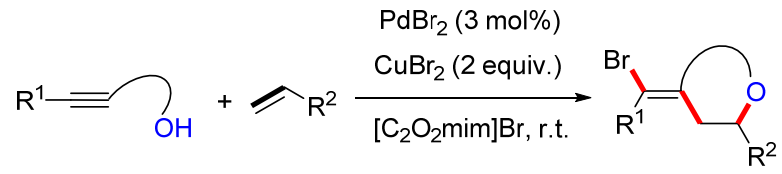

\section{1 结果与讨论}

\section{1 串联碳醚化反应的条件优化}

为了得到优化的反应条件, 实验过程中以苯基丙炔 醇(1a)与苯乙烯 (2a)为底物对串联碳醚化反应进行了探 究, 考察了离子液体的种类、催化剂的种类以及氧化剂 的种类等对反应的影响, 具体结果如表 1 所示.

在没有金属钯催化剂时, 反应是不能进行的(Entry 1). 催化剂的篎选表明(Entries $2 \sim 8$ ), 当使用 $\mathrm{PdBr}_{2}$ 作为 催化剂, 反应具有较好的气相色谱 $(\mathrm{GC})$ 转化率和较高的 选择性(Entry 3). 其他的钯催化剂对反应无明显的促进 作用, 故确定 $\mathrm{PdBr}_{2}$ 作为该条件下的最佳催化剂.

紧接着, 氧化剂的种类考察表明(Entries 3, 9 12), 当使用 2 equiv. $\mathrm{CuBr}_{2}$ 作氧化剂时, 反应有较好的转化 率和较高的选择性. 其他种类氧化剂, 诸如 $\mathrm{O}_{2}, \mathrm{BQ}$, $\mathrm{DDQ}, \mathrm{AgNO}_{3}, \mathrm{Ag}_{2} \mathrm{CO}_{3}$ 以及 $\mathrm{K}_{2} \mathrm{~S}_{2} \mathrm{O}_{8}$, 反应的 $\mathrm{GC}$ 转化率 并没有得到提高.

然后，不同种类的离子液体的考察发现(Entries 3, $15 \sim 17)$, 当使用 $\left[\mathrm{C}_{2} \mathrm{O}_{2} \mathrm{mim}\right] \mathrm{Br}$ 作溶剂时, 反应有较好的 $\mathrm{GC}$ 转化率和较高的选择性(Entry 15). 其他种类的离子 液体, 如 $[\mathrm{Bmim}] \mathrm{Br} 、[\mathrm{Bmim}] \mathrm{BF}_{4}$ 以及 $\left[\mathrm{C}_{2} \mathrm{OHmim}\right] \mathrm{Br}$, 反 应的 $\mathrm{GC}$ 转化率和选择性并没有提高. 故认为该条件下, $\left[\mathrm{C}_{2} \mathrm{O}_{2} \mathrm{mim}\right] \mathrm{Br}$ 是较为合适的离子液体. 相比较, 常规溶 剂的进一步考察发现(Entries 18 20), DMF、DMSO 以 及 $\mathrm{CH}_{3} \mathrm{CN}$ 的使用, 反应的 $\mathrm{GC}$ 转化率和选择性均出现不 同程度的降低.

最后, 温度的考察表明(Entries 21, 22), 在一定程度 上升高温度, 反应的 $\mathrm{GC}$ 转化率并没有提高. 相反地, 当温度达到 $100{ }^{\circ} \mathrm{C}$ 时, 反应的 $\mathrm{GC}$ 转化率和选择性均出 现不同程度的降低, 故认为室温是较为合适的反应温
度.

表 1 苯基丙炔醇(1a)与苯乙烯 (2a)的串联碳醚化反应的条件 优化 ${ }^{a}$

Table 1 Optimization of conditions for the cascade carboetherification of 1a with 2a

\begin{tabular}{|c|c|c|c|c|c|}
\hline Entry & Cat. [Pd] & Oxidant & Solvent & $\begin{array}{c}\mathrm{GC} \\
\text { yield/\% }\end{array}$ & $Z / E^{b}$ \\
\hline 1 & - & $\mathrm{CuBr}_{2}$ & {$[\mathrm{Bmim}] \mathrm{Br}$} & - & - \\
\hline 2 & $\mathrm{PdCl}_{2}$ & $\mathrm{CuBr}_{2}$ & {$[\mathrm{Bmim}] \mathrm{Br}$} & 47 & $93 / 7$ \\
\hline 3 & $\mathrm{PdBr}_{2}$ & $\mathrm{CuBr}_{2}$ & {$[\mathrm{Bmim}] \mathrm{Br}$} & 56 & $95 / 5$ \\
\hline 4 & $\mathrm{Pd}(\mathrm{TFA})_{2}$ & $\mathrm{CuBr}_{2}$ & {$[\mathrm{Bmim}] \mathrm{Br}$} & 15 & $94 / 6$ \\
\hline 5 & $\mathrm{PdI}_{2}$ & $\mathrm{CuBr}_{2}$ & {$[\mathrm{Bmim}] \mathrm{Br}$} & 53 & $95 / 5$ \\
\hline 6 & $\mathrm{Pd}(\mathrm{OAc})_{2}$ & $\mathrm{CuBr}_{2}$ & {$[\mathrm{Bmim}] \mathrm{Br}$} & 38 & $95 / 5$ \\
\hline 7 & $\mathrm{Pd}\left(\mathrm{CH}_{3} \mathrm{CN}\right)_{2} \mathrm{Cl}_{2}$ & $\mathrm{CuBr}_{2}$ & {$[\mathrm{Bmim}] \mathrm{Br}$} & 35 & $94 / 6$ \\
\hline 8 & {$\left[\mathrm{Pd}\left(\eta^{3}-\mathrm{C}_{3} \mathrm{H}_{5}\right) \mathrm{Cl}\right]_{2}$} & $\mathrm{CuBr}_{2}$ & {$[\mathrm{Bmim}] \mathrm{Br}$} & Trace & - \\
\hline $9 \mathrm{I}$ & $\mathrm{PdBr}_{2}$ & - & {$[\mathrm{Bmim}] \mathrm{Br}$} & 13 & $84 / 16$ \\
\hline $10 \mathrm{I}$ & $\mathrm{PdBr}_{2}$ & BQ & {$[\mathrm{Bmim}] \mathrm{Br}$} & Trace & - \\
\hline $11 \mathrm{I}$ & $\mathrm{PdBr}_{2}$ & DDQ & {$[[\mathrm{Bmim}] \mathrm{Br}$} & Trace & - \\
\hline $12 \mathrm{I}$ & $\mathrm{PdBr}_{2}$ & $\mathrm{AgNO}_{3}$ & {$[\mathrm{Bmim}] \mathrm{Br}$} & 18 & $85 / 15$ \\
\hline $13 \mathrm{I}$ & $\mathrm{PdBr}_{2}$ & $\mathrm{Ag}_{2} \mathrm{CO}_{3}$ & {$[\mathrm{Bmim}] \mathrm{Br}$} & 23 & $85 / 15$ \\
\hline $14 \mathrm{I}$ & $\mathrm{PdBr}_{2}$ & $\mathrm{~K}_{2} \mathrm{~S}_{2} \mathrm{O}_{8}$ & {$[\mathrm{Bmim}] \mathrm{Br}$} & 34 & $83 / 17$ \\
\hline $15 \mathrm{I}$ & $\mathrm{PdBr}_{2}$ & $\mathrm{CuBr}_{2}$ & {$\left[\mathrm{C}_{2} \mathrm{O}_{2} \mathrm{mim}\right] \mathrm{Br}$} & $90(84)$ & $96 / 4$ \\
\hline $16 \mathrm{I}$ & $\mathrm{PdBr}_{2}$ & $\mathrm{CuBr}_{2}$ & {$\left[\mathrm{C}_{2} \mathrm{OHmim}\right] \mathrm{Br}$} & 76 & $96 / 4$ \\
\hline $17 \mathrm{I}$ & $\mathrm{PdBr}_{2}$ & $\mathrm{CuBr}_{2}$ & {$[\mathrm{Bmim}] \mathrm{BF}_{4}$} & 28 & $62 / 38$ \\
\hline $18 \mathrm{I}$ & $\mathrm{PdBr}_{2}$ & $\mathrm{CuBr}_{2}$ & DMF & 54 & $60 / 40$ \\
\hline $19 \mathrm{I}$ & $\mathrm{PdBr}_{2}$ & $\mathrm{CuBr}_{2}$ & DMSO & 25 & $56 / 44$ \\
\hline $20 \mathrm{I}$ & $\mathrm{PdBr}_{2}$ & $\mathrm{CuBr}_{2}$ & $\mathrm{CH}_{3} \mathrm{CN}$ & 79 & $81 / 19$ \\
\hline $21^{c} \mathrm{I}$ & $\mathrm{PdBr}_{2}$ & $\mathrm{CuBr}_{2}$ & {$\left[\mathrm{C}_{2} \mathrm{O}_{2} \mathrm{mim}\right] \mathrm{Br}$} & 90 & $96 / 4$ \\
\hline $22^{d} \mathrm{I}$ & $\mathrm{PdBr}_{2}$ & $\mathrm{CuBr}_{2}$ & {$\left[\mathrm{C}_{2} \mathrm{O}_{2} \mathrm{mim}\right] \mathrm{Br}$} & 87 & $90 / 10$ \\
\hline
\end{tabular}

因此，优化后较合适的反应条件为: 室温、空气氛 围下，反应溶剂为 $0.5 \mathrm{~mL}\left[\mathrm{C}_{2} \mathrm{O}_{2} \mathrm{mim}\right] \mathrm{Br}$, 催化剂为 3 $\mathrm{mol} \% \mathrm{PdBr}_{2}$, 氧化剂为 2 equiv. $\mathrm{CuBr}_{2}$.

\section{2 钯催化串联碳醚化反应底物普适性考察}

在优化条件下，我们进一步考查此催化体系的底物 普适性(表 2). 从表 2 可知对芳基炔丙醇的考察表明：当 苯环连接供电子基(比如甲基)时，反应通常都能得到良 好的收率, 并且取代基的位置对反应的收率无显著的影 响 (3c, 3d). 进一步地, 当苯环连接吸电子基 $(\mathrm{F}, \mathrm{Cl})$ 时, 反应也都能实现较高产率的转化. 对不同烯烃种类的考 察表明，苯环取代基的性质对反应无明显的影响，无论 是供电子的甲基 $(2 \mathrm{~g})$, 还是吸电子的溴原子(2i), 反应均 能实现优良产率的转化. 遗憾的是, 4-吡啶丙炔醇 $(\mathbf{1 j})$ 和 烷基烯烃(2k)在优化的条件下，未能顺利得到预期的目 标产物 
表 2 炔丙醇与烯烃的串联碳醚化反应 ${ }^{a}$

Table 2 Cascade carboetherification of alkynols with alkenes

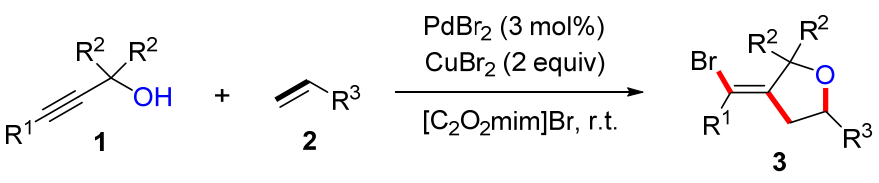<smiles>BrC(=C1COC(c2ccccc2)C1)c1ccccc1</smiles>

3a, $84 \%$

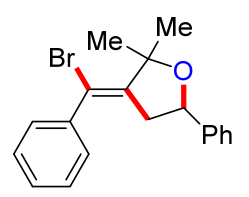

3b, $78 \%$

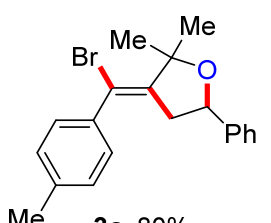

3c, $80 \%$

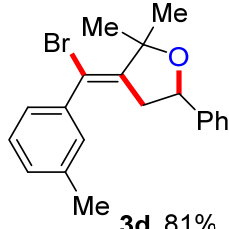

3d, $81 \%$

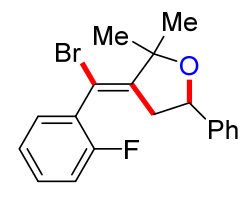

3e, $72 \%$

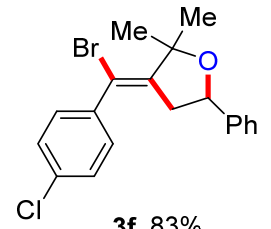

3f, $83 \%$<smiles>Cc1cccc(C2C/C(=C(/Br)c3ccccc3)C(C)(C)O2)c1</smiles>

3g, $74 \%$

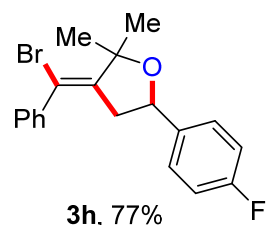

3h, $77 \%$

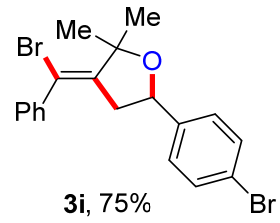

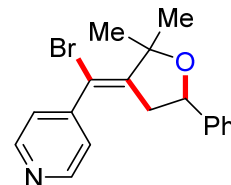

$3 \mathbf{j}$, trace

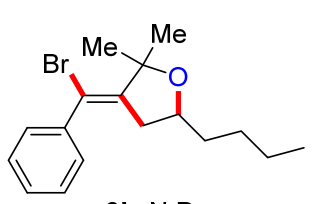

3k, N.D

${ }^{a}$ 反应条件: 1 ( $\left.0.20 \mathrm{mmol}\right), \mathbf{2}(0.30 \mathrm{mmol}), 3 \mathrm{~mol} \% \mathrm{PdBr}_{2}, 2$ equiv. $\mathrm{CuBr}_{2}, 1 \mathrm{~mL}\left[\mathrm{C}_{2} \mathrm{O}_{2} \mathrm{mim}\right] \mathrm{Br}$, 空气氛围下室温反应. 分离收率, 未特殊说明, Z/E均大于 $95: 5$.

\section{3 钯催化串联碳酯化反应底物普适性考察}

在炔丙醇与烯烃的串联碳醚化反应基础上，我们继 续考察炔酸与烯烃的串联碳酯化反应构建系列 $\alpha$-亚甲 基- $\gamma$-丁内酯化合物. 从表 3 可知, 无论芳基炔酸连接供 电子基(比如甲基), 还是吸电子基( $\mathrm{F}, \mathrm{Cl}, \mathrm{Br}, \mathrm{NO}_{2}$ )时，反 应都能实现中等到良好的转化 $(\mathbf{6 a} \sim \mathbf{6 d})$. 同时, 在优化 的条件下烷基烯烃也顺利得到预期的目标产物 $(6 \mathbf{f} \sim 6 \mathrm{~g})$.

\section{4 产物结构表征}

所得化合物的结构经 IR、 ${ }^{1} \mathrm{H}$ NMR、 ${ }^{13} \mathrm{C}$ NMR 和 HRMS 确证. 红外测试结果表明, 目标化合物在 3063 $3033 \mathrm{~cm}^{-1}$ 的较强吸收峰为 $\mathrm{C}=\mathrm{C}-\mathrm{H}$ 伸缩振动吸收峰, 波数在 $1788 \sim 1760 \mathrm{~cm}^{-1}$ 的吸收峰为 $\mathrm{C}=\mathrm{O}$ 伸缩振动吸 收峰, 波数在 $1638 \sim 1626 \mathrm{~cm}^{-1}$ 的吸收峰为 $\mathrm{C}=\mathrm{C}$ 伸缩 振动吸收峰. 分析目标化合物的 ${ }^{1} \mathrm{H}$ NMR 可知, $\delta$ 为 5.03 4.94 和 5.48 4.86 处出现的多重峰为取代的四氢 呋喃衍生物和 $\alpha$-亚甲基- $\gamma$-丁内酯 $\gamma$ 位饱和氢的化学位 移.

\section{2 结论}

本文利用易得的炔烃和烯烃为反应底物, 通过串联 氧化/杂环化反应构建系列五元氧杂环化合物. 研究结 果表明: 在 $3 \mathrm{~mol} \% \mathrm{PdBr}_{2}, 2$ equiv. $\mathrm{CuBr}_{2}, 1 \mathrm{~mL}\left[\mathrm{C}_{2} \mathrm{O}_{2}\right.$ $\operatorname{mim}] \mathrm{Br}$, 室温条件下, 各种炔丙醇和炔酸均能与烯烃衍 生物以中等至优良的产率得到目标化合物, 而且反应表 现出较好的区域选择性和立体选择性. 另外, 该反应条 件温和, 易于操作和处理, 可为具有五元氧杂环骨架的 天然产物及复杂药物分子的合成提供简便的途径.
表 3 炔酸与烯烃的串联碳酯化反应 ${ }^{a}$

Table 3 Cascade carboesterification of alkynoic acids with alkenes
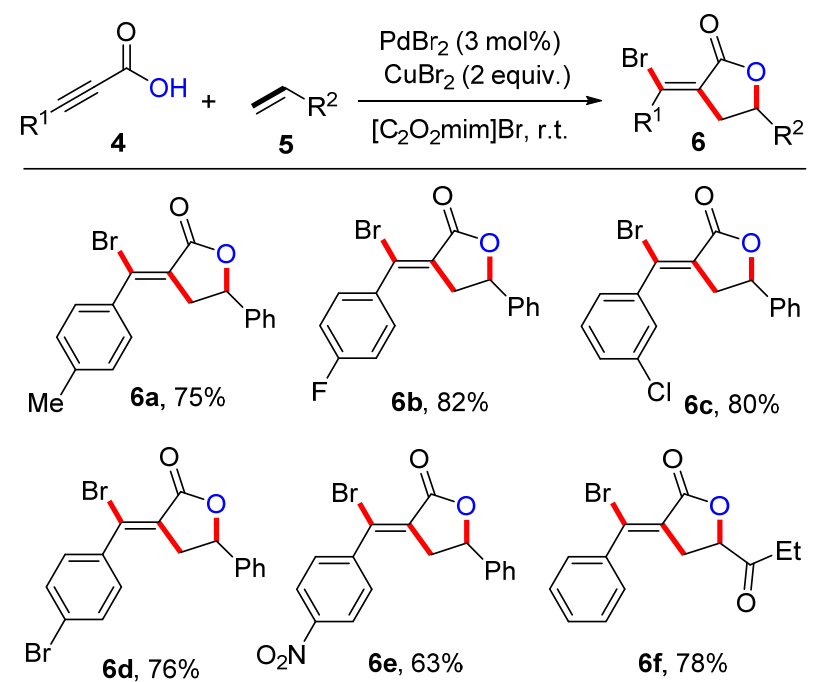<smiles>CCOC(=O)C1C/C(=C(/Br)c2ccccc2)C(=O)O1</smiles>

$6 \mathbf{g}, 80 \%$

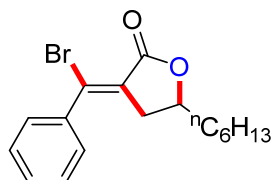

6h, $73 \%$
${ }^{a}$ 反应条件: 4 (0.2 mmol), 5 (0.3 mmol), $3 \mathrm{~mol} \% \mathrm{PdBr}_{2}, 2$ equiv. $\mathrm{CuBr}_{2}, 1 \mathrm{~mL}$ $\left[\mathrm{C}_{2} \mathrm{O}_{2} \mathrm{mim}\right] \mathrm{Br}$, 空气氛围下室温反应. 分离收率, 未特殊说明, $Z / E$ 均大于 $95 / 5$

\section{3 实验部分}

\section{1 仪器与试剂}

美国 PERKIN-ELIMER 1730 FT-IR 红外光谱仪, $\mathrm{KBr}$ 压片; 德国 Bruker 公司 $400 \mathrm{MHz}$ DRX-400 核磁共 
振仪, 溶剂 $\mathrm{CDCl}_{3}$, 内标 TMS; 美国 LCQ Deca XP MAX 液相色谱一质谱联用仪(或美国 FINNIGAN TRACE DSQ 质谱仪, $70 \mathrm{eV}$ ); 河南省予华仪器有限公司 X-5 型显微 熔点仪.

所有试剂均为市售分析纯, 未经进一步纯化. 离子 液体 $\left([\mathrm{Bmim}] \mathrm{Br}^{[18]},\left[\mathrm{Bmim}^{\left[\mathrm{BF}_{4}\right.}{ }^{[19]},\left[\mathrm{C}_{2} \mathrm{OHmim}\right] \mathrm{Br}^{[20]},\left[\mathrm{C}_{2}-\right.\right.\right.$ $\left.\mathrm{O}_{2} \operatorname{mim}\right] \mathrm{Br}^{[21]}$ )参考相应文献合成.

\section{2 实验方法}

$25 \mathrm{~mL}$ 烧瓶中加入 $0.2 \mathrm{mmol}$ 炔烃 1 或 4, $0.3 \mathrm{mmol}$ 烯烃衍生物 2 或 $5,3 \mathrm{~mol} \% \mathrm{PdBr}_{2}, 1 \mathrm{~mL}$ 离子液体 $\left[\mathrm{C}_{2} \mathrm{O}_{2} \mathrm{mim}\right] \mathrm{Br}$, 空气氛围下室温磁力摚拌, 薄层色谱 (TLC) 跟踪. 反应结束后, 往溶液中加入 $10 \mathrm{~mL}$ 乙酸乙 酯 $(10 \mathrm{~mL} \times 3)$, 萃取, 有机层无水硫酸镁干燥, 减压旋 干得粗品, 柱层析得 3 和 $\mathbf{6}$. 目标化合物的结构经 IR, ${ }^{1} \mathrm{HNMR},{ }^{13} \mathrm{C}$ NMR 和 HRMS 确证.

(Z)-4-溴(苯基)亚甲基-2-苯基四氢呋喃(3a): 黄色液 体, 产率 $84 \%$ (52.7 mg), $R_{\mathrm{f}}=0.42$ [ $V$ (石油醚)：V(乙酸乙 酯 $)=100: 1] .{ }^{1} \mathrm{H}$ NMR $\left(400 \mathrm{MHz}, \mathrm{CDCl}_{3}\right) \delta: 7.50(\mathrm{~d}, J=$ $8.0 \mathrm{~Hz}, 2 \mathrm{H}), 7.44 \sim 7.30(\mathrm{~m}, 8 \mathrm{H}), 5.03(\mathrm{dd}, J=9.2,5.6 \mathrm{~Hz}$, $1 \mathrm{H}), 4.89(\mathrm{~d}, J=14.8 \mathrm{~Hz}, 1 \mathrm{H}), 4.69$ (d, $J=14.8 \mathrm{~Hz}, 1 \mathrm{H})$, 3.08 (dd, $J=15.6,5.2 \mathrm{~Hz}, 1 \mathrm{H}), 2.78(\mathrm{dd}, J=15.6,9.2 \mathrm{~Hz}$, $1 \mathrm{H}) ;{ }^{13} \mathrm{C}$ NMR $\left(100 \mathrm{MHz}, \mathrm{CDCl}_{3}\right) \delta: 140.8,138.3,137.8$, $128.5,128.6,128.2,127.9,127.8,125.8,121.9,82.3,72.5$, 41.5; IR (KBr) v: 3056, 2936, 1780, 1628, 1506, 1425, 1126, $698 \mathrm{~cm}^{-1}$; HRMS (ESI) calcd for $\mathrm{C}_{17} \mathrm{H}_{16} \mathrm{BrO}[\mathrm{M}+$ $\mathrm{H}]^{+}:$315.0379, found 315.0376 .

(Z)-3-溴(苯基)亚甲基-2,2'-二甲基-5-苯基四氢呋喃 (3b): 黄色液体, 产率 $78 \%(53.4 \mathrm{mg}), R_{\mathrm{f}}=0.44$ [ $V$ (石油 醚) $: V($ 乙酸乙酯 $)=100 ： 1] .{ }^{1} \mathrm{H}$ NMR $(400 \mathrm{MHz}$, $\left.\mathrm{CDCl}_{3}\right) \delta: 7.48(\mathrm{~d}, J=7.6 \mathrm{~Hz}, 2 \mathrm{H}), 7.44 \sim 7.30(\mathrm{~m}, 8 \mathrm{H})$, 4.99 (dd, $J=10.6,2.8 \mathrm{~Hz}, 1 \mathrm{H}), 2.81$ (dd, $J=16.8,10.6 \mathrm{~Hz}$, $1 \mathrm{H}), 2.63(\mathrm{dd}, J=16.8,3.2 \mathrm{~Hz}, 1 \mathrm{H}), 1.66(\mathrm{~s}, 6 \mathrm{H}) ;{ }^{13} \mathrm{C}$ NMR $\left(100 \mathrm{MHz}, \mathrm{CDCl}_{3}\right) \delta: 141.6,139.9,133.5,131.8$, 128.4, 128.2, 128.1, 127.7, 127.4, 126.1, 70.3, 40.9, 28.5, 24.3; IR (KBr) v: 3048, 2932, 1776, 1625, 1443, 1415, 1360, 1126, 1024, $687 \mathrm{~cm}^{-1}$; HRMS (ESI) calcd for $\mathrm{C}_{19} \mathrm{H}_{20} \mathrm{BrO}[\mathrm{M}+\mathrm{H}]^{+}: 343.0692$, found 343.0688 .

(Z)-3-溴(4-甲基苯基)亚甲基-2,2'-二甲基-5-苯基四 氢呋喃(3c): 黄色液体, 产率 $80 \%(56.9 \mathrm{mg}), R_{\mathrm{f}}=0.45$ [ $V($ 石油醚 $) ： V($ 乙酸乙酯 $)=100 ： 1] .{ }^{1} \mathrm{H}$ NMR $(400$ $\left.\mathrm{MHz}, \mathrm{CDCl}_{3}\right) \delta: 7.46(\mathrm{~d}, J=7.6 \mathrm{~Hz}, 2 \mathrm{H}), 7.39$ (t, $J=7.6$ $\mathrm{Hz}, 2 \mathrm{H}), 7.32(\mathrm{~d}, J=7.2 \mathrm{~Hz}, 1 \mathrm{H}), 7.23$ (q, $J=7.6 \mathrm{~Hz}$, 4H), 4.96 (dd, $J=10.6,2.4 \mathrm{~Hz}, 1 \mathrm{H}), 2.77$ (dd, $J=16.8$, $10.6 \mathrm{~Hz}, 1 \mathrm{H}), 2.60$ (dd, $J=16.8,2.8 \mathrm{~Hz}, 1 \mathrm{H}), 2.39$ (s, 3H), $1.63(\mathrm{~s}, 6 \mathrm{H}) ;{ }^{13} \mathrm{C}$ NMR $\left(100 \mathrm{MHz}, \mathrm{CDCl}_{3}\right) \delta: 141.7,137.2$,
$136.9,133.1,131.7,128.9,128.5,128.1,127.7,126.0$, 77.3, 70.3, 40.9, 28.6, 24.3, 21.3; IR (KBr) v: 3045, 2924, 1781, 1626, 1500, 1427, 1328, 1122, $688 \mathrm{~cm}^{-1}$; HRMS (ESI) calcd for $\mathrm{C}_{20} \mathrm{H}_{22} \mathrm{BrO}[\mathrm{M}+\mathrm{H}]^{+}: 357.0849$, found 357.0843 .

(Z)-3-澳(3-甲基苯基)亚甲基-2,2'-二甲基-5-苯基四 氢呋喃(3d): 黄色液体, 产率 $81 \%(57.7 \mathrm{mg}), R_{\mathrm{f}}=0.45$ [ $V($ 石油醚 $) ： V($ 乙酸乙酯 $)=100 ： 1] .{ }^{1} \mathrm{H}$ NMR $(400$ $\left.\mathrm{MHz}, \mathrm{CDCl}_{3}\right) \delta: 7.48(\mathrm{~d}, J=7.6 \mathrm{~Hz}, 2 \mathrm{H}), 7.41$ (t, $J=7.2$ $\mathrm{Hz}, 2 \mathrm{H}), 7.32$ (dd, $J=16.0,8.0 \mathrm{~Hz}, 2 \mathrm{H}), 7.20 \sim 7.14(\mathrm{~m}$, $3 \mathrm{H}), 5.06 \sim 4.90(\mathrm{~m}, 1 \mathrm{H}), 2.80$ (dd, $J=16.8,10.6 \mathrm{~Hz}, 1 \mathrm{H})$, $2.62(\mathrm{dd}, J=16.8,2.4 \mathrm{~Hz}, 1 \mathrm{H}), 2.41$ (s, 3H), 1.65 (s, 6H); ${ }^{13} \mathrm{C}$ NMR $\left(100 \mathrm{MHz}, \mathrm{CDCl}_{3}\right) \delta: 141.7,139.9,137.8$, $133.3,132.0,128.7,128.5,128.2,128.1,127.7,126.1$, 125.2, 77.4, 70.3, 41.0, 28.6, 24.3, 21.5; IR (KBr) v: 3046, 2933, 1783, 1608, 1511, 1430, 1226, 1126, 1082, 698 $\mathrm{cm}^{-1}$; HRMS (ESI) calcd for $\mathrm{C}_{20} \mathrm{H}_{22} \mathrm{BrO}[\mathrm{M}+\mathrm{H}]^{+}$: 357.0849 , found 357.0845 .

(Z)-3-溴(2-氟苯基)亚甲基-2,2'-二甲基-5-苯基四氢 呋喃(3e): 黄色固体, 产率 72\% (51.8 mg). m.p. 84.3 $85.7{ }^{\circ} \mathrm{C}, R_{\mathrm{f}}=0.39$ [ $V$ (石油醚) $: V($ 乙酸乙酯 $)=100: 1$ ]. ${ }^{1} \mathrm{H}$ NMR $\left(400 \mathrm{MHz}, \mathrm{CDCl}_{3}\right) \delta: 7.47(\mathrm{~d}, J=7.6 \mathrm{~Hz}, 2 \mathrm{H})$, 7.39 (t, $J=7.6 \mathrm{~Hz}, 2 \mathrm{H}), 7.31$ (dd, $J=14.0,6.8 \mathrm{~Hz}, 3 \mathrm{H})$, $7.15(\mathrm{dt}, J=18.4,8.4 \mathrm{~Hz}, 2 \mathrm{H}), 5.08 \sim 4.91(\mathrm{~m}, 1 \mathrm{H}), 2.77$ (dd, $J=16.8,10.6 \mathrm{~Hz}, 1 \mathrm{H}), 2.58$ (dd, $J=16.8,1.6 \mathrm{~Hz}, 1 \mathrm{H}$ ), $1.65(\mathrm{~s}, 6 \mathrm{H}) ;{ }^{13} \mathrm{C}$ NMR $\left(100 \mathrm{MHz}, \mathrm{CDCl}_{3}\right) \delta: 159.4(\mathrm{~d}, J=$ $246.8 \mathrm{~Hz}), 141.5,135.8,130.4(\mathrm{~d}, J=3.8 \mathrm{~Hz}), 129.5$, 129.4, 128.4, 127.7, 127.3, 126.1, 123.9 (d, $J=3.5 \mathrm{~Hz})$, 115.8 (d, $J=21.9 \mathrm{~Hz}), 77.4,70.2,39.9,28.5,24.3$; IR (KBr) v: 3047, 2923, 1780, 1624, 1487, 1366, 1126, 697 $\mathrm{cm}^{-1}$; HRMS (ESI) calcd for $\mathrm{C}_{19} \mathrm{H}_{19} \mathrm{BrFO}[\mathrm{M}+\mathrm{H}]^{+}$: 361.0598 , found 361.0594 .

(Z)-3-澳(4-氯苯基)亚甲基-2,2'-二甲基-5-苯基四氢 呋喃(3f): 黄色固体, 产率 83\% (62.4 mg). m.p. 96.6 $98.3{ }^{\circ} \mathrm{C} ; R_{\mathrm{f}}=0.38$ [ $V$ (石油醚) $: V($ 乙酸乙酯 $)=100: 1$ ] ${ }^{1} \mathrm{H}$ NMR (400 MHz, $\left.\mathrm{CDCl}_{3}\right) \delta: 7.49$ (d, $\left.J=7.6 \mathrm{~Hz}, 2 \mathrm{H}\right)$, 7.38 (ddd, $J=24.8,16.4,8.0 \mathrm{~Hz}, 7 \mathrm{H}$ ), 4.99 (d, $J=10.6 \mathrm{~Hz}$, $1 \mathrm{H}), 2.80(\mathrm{dd}, J=16.8,10.6 \mathrm{~Hz}, 1 \mathrm{H}), 2.60(\mathrm{dd}, J=16.8$, $2.0 \mathrm{~Hz}, 1 \mathrm{H}), 1.67(\mathrm{~s}, 6 \mathrm{H}) ;{ }^{13} \mathrm{C} \mathrm{NMR}\left(100 \mathrm{MHz}, \mathrm{CDCl}_{3}\right) \delta$ : $141.5,138.2,134.2,133.3,130.8,129.6,128.5,128.4$, $127.8,126.0,77.4,70.3,40.8,28.5,24.3$; IR (KBr) $v$ : 3052, 2946, 1788, 1607, 1501, 1449, 1366, 1126, 1028, $698 \mathrm{~cm}^{-1}$; HRMS (ESI) calcd for $\mathrm{C}_{19} \mathrm{H}_{19} \mathrm{BrClO}[\mathrm{M}+\mathrm{H}]^{+}$: 377.0302 , found 377.0301 .

(Z)-3-溴(苯基)亚甲基-2,2'-二甲基-5-(3-甲基苯基) 
四氢呋喃(3g): 黄色液体, 产率 $74 \%(52.7 \mathrm{mg}), R_{\mathrm{f}}=0.43$ [ $V($ 石油醚 $): V($ 乙酸乙酯 $)=100 ： 1] .{ }^{1} \mathrm{H}$ NMR $(400$ $\left.\mathrm{MHz}, \mathrm{CDCl}_{3}\right) \delta: 7.47 \sim 7.24(\mathrm{~m}, 8 \mathrm{H}), 7.15(\mathrm{~d}, J=6.8 \mathrm{~Hz}$, $1 \mathrm{H}), 5.17 \sim 4.78(\mathrm{~m}, 1 \mathrm{H}), 2.81(\mathrm{dd}, J=16.8,10.6 \mathrm{~Hz}, 1 \mathrm{H})$, $2.62(\mathrm{dd}, J=16.8,3.0 \mathrm{~Hz}, 1 \mathrm{H}), 2.41$ (s, 3H), 1.66 (s, 6H); ${ }^{13} \mathrm{C}$ NMR $\left(100 \mathrm{MHz}, \mathrm{CDCl}_{3}\right) \delta: 141.5,139.9,138.2,133.5$, $131.9,128.5,128.4,128.2,128.1,127.4,126.8,123.2$, 77.4, 70.4, 40.9, 28.6, 24.3, 21.5; IR (KBr) v: 3060, 2924, 1763, 1638, 1504, 1449, 1124, $697 \mathrm{~cm}^{-1}$; HRMS (ESI) calcd for $\mathrm{C}_{20} \mathrm{H}_{22} \mathrm{BrO}[\mathrm{M}+\mathrm{H}]^{+}: 357.0849$, found 357.0844.

(Z)-3-澳(苯基)亚甲基-2,2'-二甲基-5-(4-氟苯基)四 氢呋喃(3h): 黄色液体, 产率 $77 \%(55.4 \mathrm{mg}), R_{\mathrm{f}}=0.38$ [ $V($ 石油醚 $): V($ 乙酸乙酯 $)=100 ： 1] .{ }^{1} \mathrm{H}$ NMR $(400$ $\left.\mathrm{MHz}, \mathrm{CDCl}_{3}\right) \delta: 7.43(\mathrm{dd}, J=15.6,8.0 \mathrm{~Hz}, 4 \mathrm{H}), 7.39 \sim$ 7.32 (m, 3H), 7.09 (t, $J=8.4 \mathrm{~Hz}, 2 \mathrm{H}), 4.96$ (dd, $J=10.6$, $2.0 \mathrm{~Hz}, 1 \mathrm{H}), 2.77$ (dd, $J=16.8,10.6 \mathrm{~Hz}, 1 \mathrm{H}), 2.61$ (dd, $J=$ 16.8, $2.4 \mathrm{~Hz}, 1 \mathrm{H}), 1.65(\mathrm{~s}, 6 \mathrm{H}) ;{ }^{13} \mathrm{C}$ NMR $(100 \mathrm{MHz}$, $\left.\mathrm{CDCl}_{3}\right) \delta: 162.3(\mathrm{~d}, J=245.6 \mathrm{~Hz}), 139.8,137.5$ (d, $J=3.1$ $\mathrm{Hz}), 133.4,131.7,128.2,128.1,127.7(\mathrm{~d}, J=8.1 \mathrm{~Hz})$, 127.5, 115.3 (d, $J=21.4 \mathrm{~Hz}), 77.5,69.7,40.9,28.5,24.3$; IR (KBr) v: 3036, 2964, 1776, 1616, 1498, 1454, 1368, 1129, 1070, $699 \mathrm{~cm}^{-1}$; HRMS (ESI) calcd for $\mathrm{C}_{19} \mathrm{H}_{19} \mathrm{BrF}-$ $\mathrm{O}[\mathrm{M}+\mathrm{H}]^{+}: 361.0598$, found 361.0593 .

(Z)-3-溴(苯基)亚甲基-2,2'-二甲基-5-(4-溴苯基)四 氢呋喃(3i): 黄色固体, 产率 75\% (63.1 mg). m.p. 87.2 $88.7{ }^{\circ} \mathrm{C} ; R_{\mathrm{f}}=0.37$ [ $V$ (石油醚) $: V$ (乙酸乙酯 $)=100: 1$ ]. ${ }^{1} \mathrm{H}$ NMR $\left(400 \mathrm{MHz}, \mathrm{CDCl}_{3}\right) \delta: 7.53(\mathrm{~d}, J=8.0 \mathrm{~Hz}, 2 \mathrm{H})$, $7.46 \sim 7.32(\mathrm{~m}, 7 \mathrm{H}), 4.94(\mathrm{dd}, J=10.4,2.4 \mathrm{~Hz}, 1 \mathrm{H}), 2.74$ (dd, $J=16.8,10.6 \mathrm{~Hz}, 1 \mathrm{H}), 2.61$ (dd, $J=16.8,2.8 \mathrm{~Hz}, 1 \mathrm{H})$, $1.65(\mathrm{~d}, J=4.8 \mathrm{~Hz}, 6 \mathrm{H}) ;{ }^{13} \mathrm{C}$ NMR $\left(100 \mathrm{MHz}, \mathrm{CDCl}_{3}\right) \delta$ : $140.7,139.7,133.5,131.6,131.6,128.3,128.1,127.8$, $127.5,121.5,77.5,69.7,40.8,28.5,24.3$; IR (KBr) $v$ : 3032, 2928, 1786, 1644, 1510, 1454, 1173, 1026, 698 $\mathrm{cm}^{-1}$; HRMS (ESI) calcd for $\mathrm{C}_{19} \mathrm{H}_{19} \mathrm{Br}_{2} \mathrm{O}[\mathrm{M}+\mathrm{H}]^{+}$: 420.9797, found 420.9793 .

(Z)-3-溴(4-甲基苯基)亚甲基-5-苯基-二氢-2(3H)-呋 喃酮 (6a): 黄色液体, 产率 $75 \%(51.3 \mathrm{mg}), R_{\mathrm{f}}=0.40$ [ $V($ 石油醚 $): V($ 乙酸乙酯 $)=100 ： 1] .{ }^{1} \mathrm{H}$ NMR $(400$ $\left.\mathrm{MHz}, \mathrm{CDCl}_{3}\right) \delta: 7.45 \sim 7.31(\mathrm{~m}, 7 \mathrm{H}), 7.25(\mathrm{~d}, J=7.6 \mathrm{~Hz}$, $2 \mathrm{H}), 5.41(\mathrm{t}, J=7.2 \mathrm{~Hz}, 1 \mathrm{H}), 3.48(\mathrm{dd}, J=16.6,7.6 \mathrm{~Hz}$, $1 \mathrm{H}), 3.08(\mathrm{dd}, J=16.6,7.2 \mathrm{~Hz}, 1 \mathrm{H}), 2.41(\mathrm{~s}, 3 \mathrm{H}) ;{ }^{13} \mathrm{C}$ NMR $\left(100 \mathrm{MHz}, \mathrm{CDCl}_{3}\right) \delta: 167.5,140.8,140.3,139.4$, 134.7, 129.2, 128.9, 128.6, 128.2, 125.4, 121.7, 76.8, 40.1, 21.4; IR (KBr) v: 3033, 2926, 1758, 1627, 1453, 1196, 697 $\mathrm{cm}^{-1}$; HRMS (ESI) calcd for $\mathrm{C}_{18} \mathrm{H}_{16} \mathrm{BrO}_{2}[\mathrm{M}+\mathrm{H}]^{+}$:
343.0328, found 343.0326 .

(Z)-3-澳(4-氟苯基)亚甲基-5-苯基-二氢-2(3H)-呋喃 酮(6b): 黄色固体, 产率 $82 \%$ (56.8 mg), m.p. 98.2 $99.8{ }^{\circ} \mathrm{C} ; R_{\mathrm{f}}=0.35$ [ $V$ (石油梄) $： V($ 乙酸乙酯 $)=5: 1$ ] $;{ }^{1} \mathrm{H}$ NMR (400 MHz, $\left.\mathrm{CDCl}_{3}\right) \delta: 7.50(\mathrm{dd}, J=8.0,5.4 \mathrm{~Hz}, 2 \mathrm{H})$, 7.34 (ddd, $J=19.6,14.0,7.6 \mathrm{~Hz}, 5 \mathrm{H}$ ), 7.10 (t, $J=8.4 \mathrm{~Hz}$, $2 \mathrm{H}), 5.40$ (t, $J=7.2 \mathrm{~Hz}, 1 \mathrm{H}), 3.43$ (dd, $J=16.6,7.2 \mathrm{~Hz}$, $1 \mathrm{H}), 3.03(\mathrm{dd}, \quad J=16.6,7.2 \mathrm{~Hz}, 1 \mathrm{H}) ;{ }^{13} \mathrm{C}$ NMR $(100$ $\left.\mathrm{MHz}, \mathrm{CDCl}_{3}\right) \delta: 167.1,163.4(\mathrm{~d}, J=252.3 \mathrm{~Hz}), 139.2$, 138.8, 133.7 (d, $J=3.4 \mathrm{~Hz}), 130.5$ (d, $J=8.7 \mathrm{~Hz}), 128.9$, 128.7, 125.3, 122.6, 115.8 (d, $J=22.0 \mathrm{~Hz}$ ), 76.8, 39.9; IR (KBr) v: 3036, 2934, 1760, 1646, 1414, 1228, 1126,1033, $697 \mathrm{~cm}^{-1}$; HRMS (ESI) calcd for $\mathrm{C}_{17} \mathrm{H}_{13} \mathrm{BrFO}_{2}[\mathrm{M}+\mathrm{H}]^{+}$: 347.0077 , found 347.0074 .

(Z)-3-澳(3-氯苯基)亚甲基-5-苯基-二氢-2 $(3 H)$-呋喃 酮(6c): 黄色固体, 产率 80\% (57.9 mg). m.p. 102.4 $103.7{ }^{\circ} \mathrm{C} ; R_{\mathrm{f}}=0.35$ [ $V$ (石油醚) $: V($ 乙酸乙酯 $)=100 ： 1$ ] . ${ }^{1} \mathrm{H}$ NMR $\left(400 \mathrm{MHz}, \mathrm{CDCl}_{3}\right) \delta: 7.50(\mathrm{~s}, 1 \mathrm{H}), 7.45 \sim 7.36$ (m, 6H), $7.33(\mathrm{~d}, J=7.6 \mathrm{~Hz}, 2 \mathrm{H}), 5.44(\mathrm{t}, J=7.2 \mathrm{~Hz}, 1 \mathrm{H})$, 3.46 (dd, $J=16.8,7.2 \mathrm{~Hz}, 1 \mathrm{H}), 3.06$ (dd, $J=16.8,7.2 \mathrm{~Hz}$, $1 \mathrm{H}) ;{ }^{13} \mathrm{C}$ NMR $\left(100 \mathrm{MHz}, \mathrm{CDCl}_{3}\right) \delta: 166.9,139.3,139.0$, $138.2,134.7,130.4,129.9,128.9,128.8,128.2,126.3$, 125.4, 123.5, 76.9, 39.8; IR (KBr) v: 3036, 2925, 1747, 1638, 1505, 1424, 1166, 997, $698 \mathrm{~cm}^{-1}$; HRMS (ESI) calcd for $\mathrm{C}_{17} \mathrm{H}_{13} \mathrm{BrClO}_{2}[\mathrm{M}+\mathrm{H}]^{+}$: 362.9782 , found 362.9778 .

(Z)-3-澳(4-溴苯基)亚甲基-5-苯基-二氢-2(3H)-呋喃 酮(6d): 黄色固体, 产率 76\% (61.7 mg). m.p. 135.8 $137.4{ }^{\circ} \mathrm{C} ; R_{\mathrm{f}}=0.33$ [ $V$ (石油醚) $: V($ 乙酸乙酯 $)=100 ： 1$ ]. ${ }^{1} \mathrm{H}$ NMR $\left(400 \mathrm{MHz}, \mathrm{CDCl}_{3}\right) \delta: 7.58(\mathrm{~d}, J=8.4 \mathrm{~Hz}, 2 \mathrm{H})$, $7.44 \sim 7.36(\mathrm{~m}, 5 \mathrm{H}), 7.33(\mathrm{~d}, J=7.6 \mathrm{~Hz}, 2 \mathrm{H}), 5.43(\mathrm{t}, J=$ $7.2 \mathrm{~Hz}, 1 \mathrm{H}), 3.44$ (dd, $J=16.6,7.6 \mathrm{~Hz}, 1 \mathrm{H}), 3.05$ (dd, $J=$ 16.6, 7.2 Hz, 1H); ${ }^{13} \mathrm{C}$ NMR $\left(100 \mathrm{MHz}, \mathrm{CDCl}_{3}\right) \delta: 167.0$, $139.1,138.7,136.5,131.9), 129.7,128.9,128.7,125.3$, 124.8, 123.0, 76.8, 39.9; IR (KBr) v: 3035, 2929, 1767, 1628, 1506, 1416, 1126, 1070, $696 \mathrm{~cm}^{-1}$; HRMS (ESI) calcd for $\mathrm{C}_{17} \mathrm{H}_{13} \mathrm{Br}_{2} \mathrm{O}_{2}[\mathrm{M}+\mathrm{H}]^{+}$: 406.9277, found 406.9272 .

(Z)-3-澳(4-硝基苯基)亚甲基-5-苯基-二氢-2(3H)-呋 喃酮(6e): 黄色固体, 产率 63\% (46.9 mg). m.p. 116.6 $117.8{ }^{\circ} \mathrm{C} ; R_{\mathrm{f}}=0.43$ [ $V$ (石油醚) $: V($ 乙酸乙酯 $)=100 ： 1$ ] ${ }^{1} \mathrm{H}$ NMR $\left(400 \mathrm{MHz}, \mathrm{CDCl}_{3}\right) \delta: 8.31(\mathrm{~d}, J=8.4 \mathrm{~Hz}, 2 \mathrm{H})$, $7.70(\mathrm{~d}, J=8.4 \mathrm{~Hz}, 2 \mathrm{H}), 7.49 \sim 7.36(\mathrm{~m}, 3 \mathrm{H}), 7.33$ (d, $J=$ $7.2 \mathrm{~Hz}, 2 \mathrm{H}), 5.48(\mathrm{t}, J=7.2 \mathrm{~Hz}, 1 \mathrm{H}), 3.45(\mathrm{dd}, J=16.8,7.2$ $\mathrm{Hz}, 1 \mathrm{H}), 3.07$ (dd, $J=16.8,7.2 \mathrm{~Hz}, 1 \mathrm{H}) ;{ }^{13} \mathrm{C}$ NMR $(100$ 
$\left.\mathrm{MHz}, \mathrm{CDCl}_{3}\right) \delta: 166.5,148.4,143.4,138.7,137.0,129.3$, 129.0, 128.9, 125.2, 124.9, 123.9, 76.8, 39.7; IR (KBr) v: 3036, 2924, 1764, 1628, 1438, 1203, 1122, $697 \mathrm{~cm}^{-1}$; HRMS (ESI) calcd for $\mathrm{C}_{17} \mathrm{H}_{13} \mathrm{BrNO}_{4}[\mathrm{M}+\mathrm{H}]^{+}: 374.0022$, found 374.0018 .

(Z)-3-溴(苯基)亚甲基-5-丙酰基-二氢-2(3H)-呋喃酮 (6f): 黄色液体, 产率 78\% (48.1 mg), $R_{\mathrm{f}}=0.36$ [ $V($ 石油 醚) : $V($ 乙酸乙酯 $)=100 ： 1]$. ${ }^{1} \mathrm{H}$ NMR $(400 \mathrm{MHz}$, $\left.\mathrm{CDCl}_{3}\right) \delta: 7.59 \sim 7.41(\mathrm{~m}, 5 \mathrm{H}), 4.74(\mathrm{dd}, J=8.4,6.4 \mathrm{~Hz}$, $1 \mathrm{H}), 3.29$ (dd, $J=17.2,8.8 \mathrm{~Hz}, 1 \mathrm{H}), 3.15$ (dd, $J=17.2,6.0$ $\mathrm{Hz}, 1 \mathrm{H}), 2.70$ (q, $J=7.2 \mathrm{~Hz}, 2 \mathrm{H}), 1.10$ (t, $J=7.2 \mathrm{~Hz}, 3 \mathrm{H})$; ${ }^{13} \mathrm{C}$ NMR (100 MHz, $\left.\mathrm{CDCl}_{3}\right) \delta: 208.0,166.3,141.8,137.4$, $130.5,128.6,128.0,120.1,77.5,33.2,32.5$, 6.8; IR (KBr) v: 2927, 2360, 1763, 1626, 1200, 1064, $696 \mathrm{~cm}^{-1}$; HRMS (ESI) calcd for $\mathrm{C}_{14} \mathrm{H}_{14} \mathrm{BrO}_{3}[\mathrm{M}+\mathrm{H}]^{+}:$309.0121, found 309.0116 .

(Z)-4-溴(苯基)亚甲基-5-氧杂四氢呋喃-2-乙酯(6g): 黄色液体, 产率 $80 \%(51.8 \mathrm{mg}), R_{\mathrm{f}}=0.27$ [ $V$ (石油醚) : $V($ 乙酸乙酯 $)=100 ： 1]$. ${ }^{1} \mathrm{H} \mathrm{NMR}\left(400 \mathrm{MHz} \mathrm{CDCl}_{3}\right) \delta$ : $7.54 \sim 7.42(\mathrm{~m}, 5 \mathrm{H}), 4.86(\mathrm{dd}, J=8.8,4.8 \mathrm{~Hz}, 1 \mathrm{H}), 4.48 \sim$ $4.03(\mathrm{~m}, 2 \mathrm{H}), 3.40(\mathrm{dd}, J=17.0,9.0 \mathrm{~Hz}, 1 \mathrm{H}), 3.11(\mathrm{dd}, J=$ 17.0, $4.8 \mathrm{~Hz}, 1 \mathrm{H}), 1.28$ (t, $J=7.2 \mathrm{~Hz}, 3 \mathrm{H}) ;{ }^{13} \mathrm{C}$ NMR $(100$ $\left.\mathrm{MHz}, \mathrm{CDCl}_{3}\right) \delta: 169.3,166.2,141.4,137.4,130.5,128.7$, 128.0, 119.8, 71.8, 62.2, 34.4, 14.0; IR (KBr) v: 2984, 1769, 1626, 1194, 1068, $696 \mathrm{~cm}^{-1}$; HRMS (ESI) calcd for $\mathrm{C}_{14} \mathrm{H}_{14} \mathrm{BrO}_{4}[\mathrm{M}+\mathrm{H}]^{+}: 325.0070$, found 325.0068 .

(Z)-3-溴(苯基)亚甲基-5-己基-二氢-2(3H)-呋喃酮 $(6 \mathrm{~h})$ : 黄色液体, 产率 73\% (49.1 mg), $R_{\mathrm{f}}=0.51$ [ $V($ 石油 醚) $: V($ 乙酸乙酯 $)=100 ： 1]$. ${ }^{1} \mathrm{H}$ NMR $(400 \mathrm{MHz}$, $\left.\mathrm{CDCl}_{3}\right) \delta: 7.56 \sim 7.42(\mathrm{~m}, 5 \mathrm{H}), 4.46 \sim 4.33(\mathrm{~m}, 1 \mathrm{H}), 3.11$ (dd, $J=16.6,7.2 \mathrm{~Hz}, 1 \mathrm{H}), 2.71(\mathrm{dd}, J=16.6,6.8 \mathrm{~Hz}, 1 \mathrm{H})$, $1.72(\mathrm{dt}, J=9.6,7.8 \mathrm{~Hz}, 1 \mathrm{H}), 1.66 \sim 1.52(\mathrm{~m}, 1 \mathrm{H}), 1.49 \sim$ $1.19(\mathrm{~m}, 8 \mathrm{H}), 0.98 \sim 0.82(\mathrm{~m}, 3 \mathrm{H}) ;{ }^{13} \mathrm{C}$ NMR $(100 \mathrm{MHz}$, $\left.\mathrm{CDCl}_{3}\right) \delta: 167.5,139.2,137.8,130.1,128.5,128.1,123.1$, 76.3, 37.1, 35.8, 31.6, 28.9, 24.8, 22.5, 14.0; IR (KBr) v: 3063, 2929, 1758, 1630, 1443, 1202, 1093, $696 \mathrm{~cm}^{-1}$; HRMS (ESI) calcd for $\mathrm{C}_{17} \mathrm{H}_{22} \mathrm{BrO}_{2}[\mathrm{M}+\mathrm{H}]^{+}: 337.0798$, found 337.0794 .

\section{References}

[1] (a) Eftekhari-Sis, B.; Zirak, M.; Akbari, A. Chem. Rev. 2013, 113, 2958.

(b) Luo, P.; Tang, R.; Zhong, P.; Li, J. Chin. J. Org. Chem. 2009, 29, 1924 (in Chinese).

(罗培松, 汤日元, 钟平, 李金恒, 有机化学, 2009, 29, 1924.)

[2] (a) Chen, J.-R.; Hu, X.-Q.; Lu, L.-Q.; Xiao, W.-J. Chem. Rev. 2015, 115,5301 . (b) Huang, H.; Ji, X.; Wu, W.; Jiang, H. Chem. Soc. Rev. 2015, 44 , 1155 .

[3] (a) Yang, L.; Huang, H. Chem. Rev. 2015, 115, 3468.

(b) Yamamoto, Y. Chem. Soc. Rev. 2014, 43, 1575.

[4] (a) Wu, W.; Jiang, H. Acc. Chem. Res. 2012, 45, 1736. (b) Wu, W.; Jiang, H. Acc. Chem. Res. 2014, 47, 2483.

[5] (a) Su, D.; Tang, W.; Hu, Y.; Liu, Y.; Yu, S.; Ma, S.; Qu, J.; Yu, D. J. Nat. Prod. 2008, 71, 784.

(b) Chen, L.; Zhu, H.; Wang, R.; Zhou, K.; Jing, Y.; Qiu, F. J. Nat. Prod. 2008, 71, 852 .

[6] (a) Liu, G.; Lu, X. Tetrahedron Lett. 2003, 44, 127.

(b) Son, S.; Fu, G. C. J. Am. Chem. Soc. 2007, 129, 1046.

(c) Huang, L.; Jiang, H.; Qi, C.; Liu, X. J. Am. Chem. Soc. 2010, 132,17652 .

(d) Huang, H.; Ji, X.; Wu, W.; Jiang, H. Chem. Commun. 2013, 49, 3351.

(e) Oliveira, C. C.; Angnes, R. A.; Correia, C. R. D. J. Org. Chem. 2013, 78, 4373.

[7] Zhang, Q.; Xu, W.; Lu, X. J. Org. Chem. 2005, 70, 1505.

[8] Wang, Z.; Lu, X. J. Org. Chem. 1996, 61, 2254.

[9] Aurrecoechea, J.; Durana, A.; Pérez, E. J. Org. Chem. 2008, 73, 3650 .

[10] Martınez, C.; Álvarez, R.; Aurrecoechea, J. M. Org. Lett. 2009, 11, 1083.

[11] (a) Wolfe, J. P.; Rossi, M. J. Am. Chem. Soc. 2004, 126, 1620.

(b) Nakhla, J.; Kampf, J.; Wolfe, J. P. J. Am. Chem. Soc. 2006, 128, 2893.

(c) Lemen, G. S.; Giampietro, N. C.; Hay, M. B.; Wolfe, J. P. J. Org. Chem. 2009, 74, 2533.

[12] Huang, L.; Wang, Q.; Liu, X.; Jiang, H. Angew. Chem., Int. Ed. 2012, $51,5696$.

[13] Huang, L.; Wang, Q.; Wu, W.; Jiang, H. J. Org. Chem. 2014, 79, 7734.

[14] Zhang, Z.; Ouyang, L.; Wu, W.; Li, J.; Zhang, Z.; Jiang, H. J. Org. Chem. 2014, 79, 10734.

[15] Zhang, Z.; Wu, W.; Liao, J.; Li, J.; Jiang, H. Chem. Eur. J. 2015, 21,6708 .

[16] Li, J.; Yang, W.; Yang, S.; Huang, L.; Wu, W.; Sun, Y.; Jiang, H. Angew. Chem., Int. Ed. 2014, 53, 7219.

[17] (a) Li, J.; Yang, S.; Jiang, H.; Wu, W.; Zhao, J. J. Org. Chem. 2013, $78,12477$.

(b) Li, J.; Yang, S. Chin. J. Org. Chem. 2013, 33, 2407 (in Chinese). (李建晓, 杨少容, 有机化学, 2013, 33, 2407.)

(c) Li, J.; Yang, S.; Wu, W.; Jiang, H. Chem. Commun. 2014, 50, 1381.

(d) Li, J.; Li, C.; Yang, S.; Luo, W. Chin. J. Org. Chem. 2015, 35, 898 (in Chinese).

(李建晓, 李春生, 杨少容, 罗维, 有机化学, 2015, 35, 898.)

(e) Li, J.; Zhu, Z.; Yang, S.; Zhang, Z.; Wu, W.; Jiang, H. J. Org. Chem. 2015, 80, 3870.

(f) Li, J.; Zhang, Z.; Li, C.; Luo, W.; Yang, S. Chin. J. Org. Chem. 2015, 35, 2199 (in Chinese).

(李建晓, 张振明, 李春生, 罗维, 杨少容, 有机化学, 2015, 35, 2199.)

[18] Huddleston, J. G.; Willauer, H. D.; Swatloski, R. P.; Visser, A. E.; Rogers, R. D. Chem. Commun. 1998, 1765.

[19] Park, S.; Kazlauskas, R. J. J. Org. Chem. 2001, 66, 8395.

[20] Branco, L. C.; Rosa, J. N.; Ramos, J. J. M.; Afonso, C. A. M. Chem. Eur.J. 2002, 8, 3671.

[21] Miao, C. X.; He, L. N.; Wang, J. Q.; Wang, J. L. Adv. Synth. Catal. 2009, 351, 2209 .

(Li, L.; Fan, Y.) 